\title{
DNA methylation of the zinc transcriptional regulatory element and its potential contribution to zinc dyshomeostasis in ageing
}

\author{
J. E. J. Hardyman, O. A. Ogo, J. Tyson, L. A. Wakeling, R. A. Valentine and D. Ford \\ Human Nutrition Research Centre, and Institute for Cell and Molecular Biosciences, Newcastle University, \\ Newcastle Upon Tyne, NE2 $4 H H U K$
}

\begin{abstract}
An age-associated decrease in zinc status has been observed in several studies but the underlying causes remain uncertain. A decrease in zinc absorption due to changes in expression of zinc transporters may be a contributory factor. Zinc dyshomeostasis, including altered zinc transporter expression, has been associated also with several diseases of ageing, including Alzheimer's disease, prostate cancer, type-2 diabetes and macular degeneration. Mechanisms responsible for age- and disease-associated changes in zinc transporter expression are still to be uncovered.

The zinc transcriptional regulatory element (ZTRE) represses gene expression at high levels of zinc availability through the binding of a transcriptional regulatory factor, and has been shown to be functional in the SLC30A5 and SL30A10 human zinc transporter genes ${ }^{(1)}$. After identifying the ZTRE we noted that a CpG site in the SLC30A5 promoter at which we previously observed an age-related increase in methylation in human intestine ${ }^{(2)}$ is within the ZTRE. Methylation of this CpG site reduced binding of the regulatory protein, and the inhibitory effect was augmented when three additional $\mathrm{CpG}$ sites within 50 base pairs of the ZTRE were also methylated ${ }^{(1)}$.

We propose that age-related epigenetic modifications, including DNA methylation, contribute to changes in the expression of zinc transporters to affect zinc homeostasis. We propose specifically that age-associated changes in DNA methylation at CpG sites within or in close proximity to ZTREs in the promoter regions of other zinc transporter genes affect the binding of transcription factors, as observed for SLC30A5, thus contributing to impaired zinc homeostasis in the ageing gut and to zinc-related disease processes in other tissues.

Bioinformatic analysis using Fuzznuc software identified ZTREs within $1 \mathrm{~kb}$ upstream of the transcription start site of the human SLC3OA1, SLC3OA3, SLC3OA4, SLC30A7 and the mouse Slc30a4 and Slc30a5 zinc transporter genes. Using pyrosequencing, we measured DNA methylation around the ZTRE in the Slc30a5 gene in samples of young (4-8 mo; $n=6-7)$ and old (32-36 mo; $n=4)$ mouse intestine. In contrast to our findings in human samples, there was no difference in methylation of the CpG site within the Slc30a5 ZTRE or of $7 \mathrm{CpG}$ sites within 28 base pairs of this site between the two groups (Kruskal-Wallis $P=0.571$ ). Future work will include repeating these measurements on a larger number of samples and also extending the analysis to the mouse Slc30a4 gene and to human zinc transporter genes additional to SLC30A5.

These preliminary findings, in light of the established effect on protein binding to the ZTRE, indicate that age-related changes in DNA methylation at specific CpG sites within or close to ZTREs in genes with roles in zinc homeostasis may affect zinc homeostasis in older age. Should analysis of larger numbers of samples reveal robust associations between age and methylation at CpG sites within and close to ZTREs it will be essential to establish if these changes affect gene expression, regulation by zinc and their functional consequences.
\end{abstract}

1. Coneyworth LJ, Jackson KA, Tyson J et al. (2012) J Biol Chem 287, 36567-36581.

2. Coneyworth LJ, Mathers JC and Ford D (2009) Proc Nutr Soc 68, 1-6. 